\title{
A PROPOS D'EIMERIA THIANETHI GWÉLESSIANY, 1935
}

\author{
Par W.-L. YAKIMOFF
}

Les Annales de Parasitologie (1) ont publié une note de M. Gwéléssiany, dans laquelle l'auteur décrit une coccidie trouvée par lui en Georgie, dans cinq cas, deux chez des vaches et trois chez des buffles. Il prétend que cette coccidie est nouvelle et la nomme Eimeria thianethi. Selon nous, cette coccidie n'est autre qu'Eimeria smithi, décrite par nous et par notre élève M. Galouzo en 1927 et qu'E. bukidnonensis, décrite par M. Tubangui, en 1931.

M. Gwéléssiany n'a mesuré que dix oocystes et a trouvé les dimensions suivantes : $40,3-48,7 \mu \times 26,2-33,7 \mu$, en moyenne $32,6 \times 28,6 \mu$, formindex $0,53-0,77$, en moyenne 0,66 . En comparant ces chiffres avec les chiffres indiqués dans notre livre : « Les maladies des animaux domestiques à protozoaires » (1931), pour Eimeria smithi, il en résulte que la dimension moyenne de cette dernière est de : $32,6 \times 22,7 \mu$, formindex $0,67-0,71$. Cependant des corrections importantes dans ces dimensions ont été faites dans nos travaux ultérieurs, par exemple : dans le travail concernant les coccidies des bovidés et des petits ruminants en Azerbaidjan, imprimé dans les «Travaux de l'Institut scientifique d'Azerbaidjan 》 (I, fasc. 1), et ensuite dans les «Annales de la Société belge de médecine tropicale 》(XII, $\left.\mathrm{n}^{\circ} 1,1933\right)$. M. Gwéléssiany ne pouvait done ignorer qu'il existe des chiffres concernant les dimensions et le formindex d'Eimeria smithi (voir le tableau ci-joint).

N'envisageant pas les dimensions moyennes de M. Gwéléssiany ne concernant qu'une quantité insignifiante de mensurations d'oocystes (dix seulement), nous voyons que les dimensions d'Eimeria thianethi ne diffèrent en rien de celles d'Eimeria smithi. De 1935 jusqu'à maintenant, nous examinâmes plusieurs milliers de bovidés, dont nous ramassâmes les excréments dans bien des endroits de l'U.R.S.S. (depuis la Russie Blanche jusqu'à l'Extrême-Orient et depuis Archangel jusqu'aux frontières de Turquie, d'Iran, d'Afgha-

(1) Ann. parasit. hum. et comp., XIII, 1935, p. 338.

Annales de Parasitologie, T. XIV, $\mathrm{N}^{\circ} 3 .-1^{\text {er }}$ mai 1936, p. 295-297. 
nistan et de Mandchourie), et partout Eimeria smithi répond aux mensurations que nous venons de donner.

C'est ainsi qu'Eimeria thianethi ne se distingue nullement d'Eimeria smithi décrite par nous, ni par ses dimensions, ni par le formindex.

Pour les autres caractères, il n'existe pas non plus la moindre différence. Ainsi Eimeria thianethi a une forme ovale, mais Eimeria smithi, outre une forme ovoïde, a aussi quelquefois une forme ovale. Ne nous arrêtons pas à la couleur des oocystes, car Eimeria

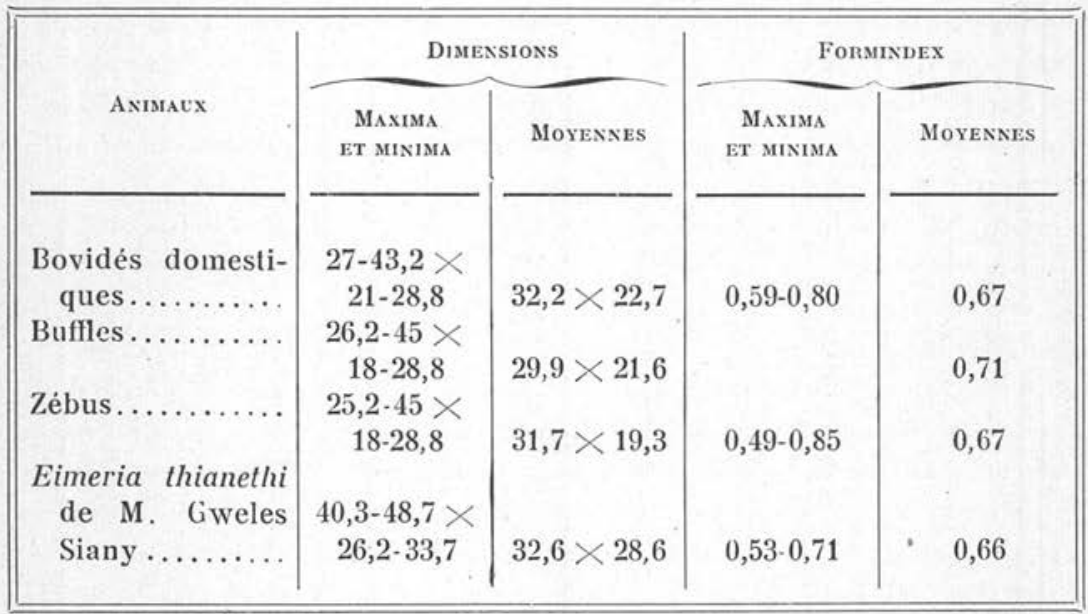

smithi aussi peut être non seulement jaunâtre, mais encore jaunâtre sale, et que M. Gwéléssiany dit que «les oocystes d'Eimeria thianethi sont de couleur variant du jaunâtre sale à l'incolore ». L'unique différence qu'on pourrait admettre entre Eimeria thianethi et $E$. smithi, c'est qu'on ne remarque jamais de striation transversale de la membrane chez Eimeria smithi, tandis que chez Eimeria thianethi elle est toujours présente. Nous croyons que dans ce cas M. Gwéléssiany confond son Eimeria thianethi avec E. bukidnonensis. En réalité, Eimeria bukidnonensis n'est pas toujours telle que M. Gwéléssiany la montre dans ses dessins (4); elle peut avoir les formes que M. Gwéléssiany représente dans son dessin 6, ce que nous avons remarqué maintes fois chez les Eimeria bukidnonensis, en revisant plusieurs centaines de mille de coccidies de bovidés, d'autant plus que les amplitudes de variation des dimensions d'Eimeria bukidnonensis sont très étendues : depuis $33 \mu$ à presque $69 \mu$. 
De cette façon, nous voyons qu'Eimeria thianethi n'a droit à aucune individualité et passe tantôt pour Eimeria smithi, tantôt pour E. bukidnonensis.

M. Gwéléssiany prétend en outre qu'il n'existe en tout, en U.R.S.S., que quatre coccidies : Eimeria smithi, E. zürni, E. ellipsoidalis et $E$. bukidnonensis, tandis qu'en réalité il y en a sept. Outre les quatre espèces mentionnées ci-dessus, il y a encore : Eimeria azerbaidschanica, E. zurnabadensis et E. cylindrica, que nous avons vues plus d'une fois. Il est vrai que M. Gwéléssiany nie l'individualité d'Eimeria zurnabadensis et d'E. azerbaidschanica, sans donner les motifs justifiant son opinion. Cependant, nous voyons des oocystes de ces coccidies non seulement en Transcaucasie, mais encore en d'autres endroits de l'Union. Mon élève M. Gousseff a vu Eimeria azerbaidschanica comme Darmpassant chez un cheval. 